\title{
FINITE AFFINE ALGEBRAS ARE FULLY DUALIZABLE
}

\author{
WOLFRAM BENTZ, PIERRE GILLIBERT, AND LUÍS SEQUEIRA
}

\begin{abstract}
We show that every finite affine algebra $\boldsymbol{A}$ admits a full duality. In the process, we prove that $\boldsymbol{A}$ also allows a strong duality, and that the duality may be induced by a dualizing structure $\underset{\sim}{A}$ of finite type. We give an explicit bound on the arities of the partial and total operations appearing in $A$. In addition, we show that the enriched partial hom-clone of $\boldsymbol{A}$ is finitely generated as a clone.
\end{abstract}

\section{INTRODUCTION}

A full duality represents elements of abstract algebraic structures by using functions on a topological space that is often enriched with a relational and/or operational structure, and vice versa. This representation allows us to solve algebraic questions by the way of the additional structure. For example in Stone duality, Boolean algebras are dual to Boolean spaces. Under this correspondence, the familiar Cantor space is dual to the denumerable free Boolean algebra, with many of the universal properties of the Cantor space being dual counterparts to the natural universal properties of being a free algebra (the universal mapping property for example).

In a natural full duality, the representation is constructed in a certain systematic way, using a generating algebra $\boldsymbol{A}$ and a corresponding topological structure $\boldsymbol{A}$, called an "alter ego" of $\boldsymbol{A}$. We say that $\boldsymbol{A}$ is fully dualizable, if there exists an alter ego $\boldsymbol{A}$ such that every algebra from the quasi-variety generated by $\boldsymbol{A}$ and every topological structure from the topological quasivariety generated by $\underset{\sim}{\boldsymbol{A}}$ has a representation. We remark that in case of a full duality, the correspondence can be extended to homomorphisms and continuous structure preserving maps, yielding a category-theoretic dual equivalence between the corresponding categories.

A full duality is the symmetrized concept of a duality. The definitions of duality and dualizability differ from that of full duality and full dualizability by requiring that only the algebras in the quasivariety generated by $\boldsymbol{A}$ have duals, while the topological quasivariety generated by $\underset{\sim}{\boldsymbol{A}}$ might contain structures without a representation.

Despite a growing understanding of duality theory, dualizability and full dualizability of an algebra continue to be mysterious properties. For some classes of algebras (such as algebras generating congruence-distributive varieties) there exists a well-behaved dividing line between the dualizable and non-dualizable algebras.

Date: June 2, 2017.

2010 Mathematics Subject Classification. 08C20, 08B10.

Key words and phrases. natural duality, strong duality, dualizable algebra, affine algebra, Abelian algebra. 
In other cases, the partial results available seem to defy any discernable pattern. This latter case includes classes of algebras that are otherwise considered to be well-understood, such as Maltsev algebras (or even extensions of groups).

Abelian algebras in congruence-modular varieties, are among the most wellbehaved classes of algebras, being polynomially equivalent to modules, hence such Abelian algebras are affine algebras. Surprisingly, most results concerning their dualizability have been announced relatively recently.

In 1995, Davey and Quackenbush [4] showed the dualizability of finite semisimple Abelian algebras from congruence-modular varieties. Recently, Kearnes and Szendrei [8] established a sufficient condition for dualizability, implying in particular the dualizability of finite affine algebras. Independently, Gillibert also proved the dualizability of finite affine algebras [5], answering a question from [1].

In this article, we will complete the remaining dualizability question for finite affine algebras by showing the following Theorem.

Theorem 1.1. All finite affine algebras are fully dualizable.

In fact we will show slightly more. Firstly, we show that a full duality can be obtained by an alter ego $\underset{\sim}{\boldsymbol{A}}$ of finite type, and we give an explicit bound on the arity of the (partial) functions and relations in $\boldsymbol{A}$. Secondly, we establish full dualizability by showing that every finite affine algebra satisfies the stronger property of (adequately named) strong dualizability.

Additionally, we obtain a structural result in clone theory. An $n$-ary partial operation $f$ over $A$ is compatible with an algebra structure $\boldsymbol{A}$ if the domain $D$ of $f$ is a subalgebra of $\boldsymbol{A}^{n}$, and $f$ is a homomorphism from $\boldsymbol{D}$ to $\boldsymbol{A}$. We show that the clone of all partial functions compatible with an affine algebra $\boldsymbol{A}$ is finitely generated as a clone (Corollary 5.4).

The proof of our main theorem relies on a technical condition from [6] (Theorem 2.7), that requires us to find a suitable factorization for each partial $\boldsymbol{A}$ compatible function through a bounded set of partial functions. Our article is structured around this requirement as follows: In Section 2 we define basic terms and establish several results about affine algebras. Section 3 provides a technical result about the factorization of projections on partial domains in the quasivariety generated by $\boldsymbol{A}$. This result will allow us to concentrate our further considerations on partial homomorphisms without proper extensions. In Section 4, we prove a crucial theorem about those partial homomorphisms: namely, a partial homomorphism that cannot be extended must have a large domain. This result is then used in Section 5 to prove a factorization property for all partial homomorphisms, and to prove our main theorem.

Section 6 and Section 7 contain an example calculation and a list of problems motivated by our research. Moreover, we have included an appendix that gives explicit bounds on the number of various algebraic objects. While the results of the appendix are used in our arguments, they are only necessary in establishing an explicit bound on the arities used in a fully dualizing alter ego. A reader without an interest in such an explicit bound may ignore the appendix and instead check the simple fact that all quantities in our argument are finite. 


\section{BASIC CONCEPTS}

Given an algebra $\boldsymbol{A}$, we denote by $A$ its underlying set, and by $\operatorname{Sub}(\boldsymbol{A})$ the set of subalgebras of $\boldsymbol{A}$. The variety $\operatorname{Var}(\boldsymbol{A})$ (respectively, the quasivariety $\mathrm{Q} \operatorname{Var}(\boldsymbol{A})$ ) generated by $\boldsymbol{A}$ is the smallest classes of algebras, with the signature of $\boldsymbol{A}$ that is closed under taking products, subalgebras, and homomorphic images (respectively, products, subalgebras, and isomorphic algebras).

For an arbitrary set $X$ and a variety $\mathcal{V}$, we denote by $F_{\mathcal{V}}(X)$ the algebra freely generated by $X$ in $\mathcal{V}$.

A subproduct algebra $\boldsymbol{A} \leq \Pi_{i} \boldsymbol{A}_{i}$ is called a subdirect product if $\pi_{i}(A)=A_{i}$ for each projection $\pi_{i}$. An algebra is subdirectly irreducible if whenever it is isomorphic to a subdirect product, it is already isomorphic to one of its factors.

An algebra $\boldsymbol{A}$ is affine if there exists an Abelian group structure $\langle A ;+, 0,-\rangle$ such that $t(x, y, z)=x-y+z$ is both a term function of $\boldsymbol{A}$ and a homomorphism from $\boldsymbol{A}^{3}$ to $\boldsymbol{A}$. Such a term $t$ is called a Maltsev term. A class of algebras $\mathcal{C}$ is affine if all of its algebras are. In the case of an affine variety $\mathcal{V}$, it is easy to see that we may choose one term $t$ that witnesses the affinity simultaneously for all members of $\mathcal{V}$ (e.g. we could take the term witnessing the affinity of $F_{\mathcal{V}}(\omega)$ ).

For example, let $\boldsymbol{G}$ be an Abelian group, and consider $t: G^{3} \rightarrow G,(x, y, z) \mapsto$ $x-y+z$. Then $(G, t)$ is an affine algebra. Or let $\boldsymbol{A}$ be an affine space over some field $\boldsymbol{F}$. For all $\lambda_{1}, \ldots, \lambda_{n}$ in $\boldsymbol{F}$ such that $\lambda_{1}+\cdots+\lambda_{n}=1$, consider the operation $f_{\left(\lambda_{1}, \ldots, \lambda_{n}\right)}: A^{n} \rightarrow A$ which maps $\left(a_{1}, \ldots, a_{n}\right)$ to the barycenter of $\left(a_{1}, \ldots, a_{n}\right)$ with weight $\left(\lambda_{1}, \ldots, \lambda_{n}\right)$. Denote by $\mathcal{P}$ the set of all such operations. Then $(A, \mathcal{P})$ is an affine algebra.

The notion of an affine algebra is closely related to that of an Abelian algebra. An algebra $\boldsymbol{A}$ is Abelian if $\left[1_{A}, 1_{A}\right]=0_{A}$, where $1_{A}$ and $0_{A}$ are the universal and trivial relations on $A$, and $[\cdot, \cdot]$ denotes the binary commutator on the congruences of $\boldsymbol{A}$ (we refer to [3] for the definition of the commutator). In congruence modular varieties, Abelian and affine algebras coincide [3, Corollary 5.9].

We repeat several results about congruences of affine algebras from [5].

Definition 2.1 ([5], Definition 3.1). Let $\boldsymbol{A}$ be an affine algebra and $\boldsymbol{B} \in \operatorname{Sub}(\boldsymbol{A})$. The congruence generated by $B$, denoted by $\Theta_{B}$ is the smallest congruence of $\boldsymbol{A}$ containing $B^{2}$.

We remark that not every congruence of $\boldsymbol{A}$ can be written in the form $\Theta_{B}$ for some subalgebra $\boldsymbol{B}$ of $\boldsymbol{A}$, and that we might have $\Theta_{B}=\Theta_{C}$ with $\boldsymbol{B} \neq \boldsymbol{C}$.

Lemma 2.2 ([5], Lemma 3.3). Let $\boldsymbol{A}$ be an affine algebra, let $\boldsymbol{B} \in \operatorname{Sub}(\boldsymbol{A})$, and let $t$ be a term witnessing the affinity of $\boldsymbol{A}$. Then

$$
\Theta_{B}=\left\{(x, y) \in A^{2} \mid \exists b \in B, t(x, y, b) \in B\right\} .
$$

Note that this result implies that $B$ is a congruence class of $\Theta_{B}$.

Lemma 2.3 ([5], Corollary 3.7). Let $\boldsymbol{A}$ be an affine algebra and let $\boldsymbol{B} \in \operatorname{Sub}(\boldsymbol{A})$ such that $B$ is meet irreducible in the semilattice $\langle\operatorname{Sub}(\boldsymbol{A}) ; \cap\rangle$. Then $\boldsymbol{A} / \Theta_{B}$ is subdirectly irreducible.

It is well known that any affine variety is polynomially equivalent to a variety of modules. Moreover, if a variety is generated by a finite affine algebra $\boldsymbol{A}$ with Maltsev term $t$, and $u \in A$, then the corresponding ring is isomorphic to the set of all unary polynomial functions of $\boldsymbol{A}$ fixing $u$, with addition defined by $f+g=t(f, u, g)$, 
and multiplication as composition of maps. In this case, the zero of the ring is the constant map with image $u$, and the unit is the identity map. Also note that any polynomial function fixing $u$ can be chosen as $x \mapsto f(x, u)$, for some idempotent binary term $f$ of $\boldsymbol{A}$.

Lemma 2.4. Let $\mathcal{V}$ be a variety generated by an affine algebra $\boldsymbol{A}$, where $|A|=$ $p_{1}^{\alpha_{1}} \ldots p_{k}^{\alpha_{k}}$ for distinct primes $p_{1}, \ldots, p_{k}$. Let $\boldsymbol{R}$ be a ring associated to $\mathcal{V}$. Then $|R|$ divides $p_{1}^{\alpha_{1}^{2}} \ldots p_{k}^{\alpha_{k}^{2}}$.

Proof. Let $u \in A$. We can assume that $\boldsymbol{R}$ is the set all unary polynomial functions of $\boldsymbol{A}$ fixing $u$, as defined before. Consider the group operation + over $A$ defined by $x+y=t(x, u, y)$ for all $x, y \in A$. Note that by construction $\langle R ;+\rangle$, is a subgroup of $\langle A ;+\rangle^{A}$. As $\boldsymbol{A}$ is an affine algebra it follows that each $f \in R$ is compatible with $t$, hence for all $x, y \in A$ the following equalities hold

$$
f(x+y)=f(t(x, u, y))=t(f(x), f(u), f(y))=t(f(x), u, f(y))=f(x)+f(y) .
$$

Therefore $\langle R ;+\rangle$ is a subgroup of $\langle\operatorname{Hom}(\langle A ;+\rangle,\langle A ;+\rangle) ;+\rangle$. By Lemma 8.1(2), $|R|$ divides $p_{1}^{\alpha_{1}^{2}} \ldots p_{k}^{\alpha_{k}^{2}}$.

Given sets $A, B$ we denote by $\mathcal{F}(A, B)$ the set of all maps $A \rightarrow B$. Let $A$ be a set. Given $n \in \mathbb{N}$ we consider the set of $n$-ary partial operations defined by:

$$
\mathfrak{C}^{n}(A)=\bigcup\left\{\mathcal{F}(X, A) \mid X \subseteq A^{n}, X \neq \emptyset\right\} .
$$

The set of all partial operations over $A$ is

$$
\mathcal{C}(A)=\{\emptyset\} \cup \bigsqcup_{n \in \mathbb{N}} \mathrm{e}^{n}(A)
$$

Note that alternative definitions distinguish empty functions of different arity; the difference is immaterial for our results. Denote by $\pi_{i}^{n}: A^{n} \rightarrow A, \vec{x} \mapsto x_{i}$ the canonical projection for all positive integers $n$ and all $1 \leq i \leq n$. A partial clone over a $A$ is a set $\mathcal{F} \subseteq \mathcal{C}(A)$, such that $\mathcal{F}$ contains all projections and is closed under composition of partial functions.

Let $\mathcal{F}$ be a partial clone over $A$. A domain of arity $n$ of $\mathcal{F}$ is a subset $D$ of $A^{n}$ such that there exists $f \in \mathcal{F}$ with $\operatorname{dom} f=D$.

Lemma 2.5. Let $\mathcal{F}$ be a partial clone over a set $A, n$ a positive integer, and $C, D$ domains of arity $n$ of $\mathcal{F}$. The following statements hold.

(1) For all $1 \leq i \leq n$, the restriction of $\pi_{i}^{n}$ to $D$ belongs to $\mathcal{F}$.

(2) The set $C \cap D$ is a domain in $\mathcal{F}$.

(3) Let $p \in \mathcal{F}$ of arity $n$. If $D \subseteq \operatorname{dom} p$, then $p\lceil D$ belongs to $\mathcal{F}$.

(4) If $p=\left(p_{1}, \ldots, p_{n}\right): A^{k} \rightarrow A^{n}$, where all $p_{i}$ are in $\mathcal{F}$, then $p^{-1}(D)$ is a domain of $\mathcal{F}$.

Proof. Take $f: C \rightarrow A$ and $g: D \rightarrow A$ in $\mathcal{F}$.

(1) is a special case of (3), shown below.

(2) $\pi_{1}^{2}(f(\vec{x}), g(\vec{x}))$ is defined if and only if $\vec{x} \in C \cap D$, thus $C \cap D$ is a domain in $\mathcal{F}$.

(3) $\pi_{1}^{2}(p(\vec{x}), g(\vec{x}))$ is defined if and only if $\vec{x} \in \operatorname{dom} p \cap \operatorname{dom} g=D$. Moreover $p(\vec{x})=\pi_{1}^{2}(p(\vec{x}), g(\vec{x}))$, for all $\vec{x} \in D$, therefore $p\lceil D$ belongs to $\mathcal{V}$.

(4) The domain of $g \circ p$ is $p^{-1}(D)$. 
We will consider partial functions whose domains are subalgebras and which are homomorphisms. For algebras $\boldsymbol{A} \leq \boldsymbol{B}$ and $\boldsymbol{C}$ and a homomorphism $f$ from $\boldsymbol{A}$ to $\boldsymbol{C}$, we say that $f$ has a proper extension if there is an algebra $\boldsymbol{D}$ with $\boldsymbol{A}<\boldsymbol{D} \leq \boldsymbol{B}$ and a homomorphism $f^{\prime}$ from $\boldsymbol{D}$ to $\boldsymbol{C}$ that extends $f$.

Our aim is to establish that all affine algebras are fully dualizable. We will now recall the definition of full dualizability and of related terms from [2]. We remark this we will not actually use any of these definitions, instead relying on established technical results to prove our claims.

For any finite algebra $\boldsymbol{A}$, consider a topological structure $\underset{\sim}{\boldsymbol{A}}=\langle A ; \mathcal{F}, \mathcal{R}, \tau\rangle$ on the universe of $\boldsymbol{A}$, where $\mathcal{F}$ is a set of (total or partial) operations, $\mathcal{R}$ is a set of relations, and $\tau$ is the discrete topology. If each fundamental operation of $\boldsymbol{A}$ preserves every relation in $\mathcal{R}$ and is compatible with every functions in $\mathcal{F}$, then $\underset{\sim}{\boldsymbol{A}}$ is called an alter ego of $\boldsymbol{A}$.

To $\boldsymbol{A}$ and $\boldsymbol{A}$ we attach two categories $\mathcal{A}, \mathcal{X}$, respectively. Here $\mathcal{A}$ consists of all algebras in $\mathbf{Q} \tilde{\operatorname{Var}}(\boldsymbol{A})$ together with their homomorphisms. The objects of $\mathcal{X}$ are all isomorphic images of (topologically) closed substructures of products of $A$, where the products are taken over non-empty index sets. The morphisms of $\mathcal{X}$ consist of all continuous mappings that preserve relations of $\mathcal{R}$ and are compatible with the operations of $\mathcal{F}$.

For $\boldsymbol{B} \in \mathcal{A}$, let $D(\boldsymbol{B}) \in \mathcal{X}$ be the substructure of $\boldsymbol{A}^{B}$ whose universe consists of all homomorphisms from $\boldsymbol{B}$ to $\boldsymbol{A}$. Reversely, for $\tilde{\mathbf{X}} \in \mathcal{X}$, let $E(\mathbf{X}) \in \mathcal{A}$ be the subalgebra of $\boldsymbol{A}^{X}$ whose universe consists of all homomorphisms from $\mathbf{X}$ to $\boldsymbol{A}$. Then $D$ and $E$ are well-defined and can be extended to contravariant functors between $\mathcal{A}$ and $\mathcal{X}$.

Now for each $\boldsymbol{B} \in \mathcal{A}$, there is a natural embedding $e_{\boldsymbol{B}}$ of $\boldsymbol{B}$ into $E D(\boldsymbol{B})$ through evaluation. That is, for all $b \in B, e_{\boldsymbol{B}}(b)$ maps $h$ to $h(b)$, for all $h \in D(\boldsymbol{B})$. A correspondingly defined embedding $\varepsilon_{\mathbf{X}}: \mathbf{X} \rightarrow D E(\mathbf{X})$ exists for all $\mathbf{X} \in X$.

We say that an alter ego $\boldsymbol{A}$ dualizes $\boldsymbol{A}$, if $e_{\boldsymbol{B}}$ is an isomorphism for each $\boldsymbol{B}$. If in addition, all $\varepsilon_{\mathbf{X}}$ are isomorphisms, we say that $\underset{\sim}{\boldsymbol{A}}$ fully dualizes $\boldsymbol{A}$. An algebra $\boldsymbol{A}$ is [fully] dualizable if there exists an alter ego that [fully] dualizes $\boldsymbol{A}$.

If $\boldsymbol{A}$ is [fully] dualizable, then $D$ and $E$ induce a dual representation [dual equivalence] between the categories $\mathcal{A}$ and $X$. The aforementioned duality between Boolean algebras and Boolean spaces can be obtained by choosing $\boldsymbol{A}$ as the two-element Boolean algebra and $\boldsymbol{A}$ as the two-element Boolean space.

Another well known related concept is that of Pontryagin duality, which can be obtained in a similar fashion by choosing $\boldsymbol{A}=\boldsymbol{A}$ to be the circle group with its usual topology. This induces a self-duality on the category of all locally compact Abelian groups and continuous homomorphisms. However, Pontryagin duality is not a direct example of our approach, as the circle group is infinite, carries a nondiscrete topology, and both $\boldsymbol{A}$ and $\boldsymbol{A}$ are topological structures.

As mentioned, we will not be using the dualizability definitions directly and instead utilize the following results from [2] and [6]. Here, strong dualizability is a special type of full dualizability that we will not define, instead referring the reader to $[2]$. 
Definition 2.6 ([6]). A finite algebra $\boldsymbol{A}$ has enough total algebraic operations, if there exists $\varphi: \omega \rightarrow \omega$ such that for all $\boldsymbol{B} \leq \boldsymbol{C} \leq \boldsymbol{A}^{n}$ and every $h \in \operatorname{hom}(\boldsymbol{B}, \boldsymbol{A})$, which has an extension to $\boldsymbol{C}$, there exists $X \subseteq \operatorname{hom}\left(\boldsymbol{A}^{n}, \boldsymbol{A}\right)$ such that

(1) $|X| \leq \varphi(|B|)$,

(2) There is a homomorphism $q$ from $\boldsymbol{C} / \cap\{\operatorname{ker}(f\lceil C) \mid f \in X\}$ to $\boldsymbol{A}$ such that $q \circ \alpha=h$, where $\alpha$ is the natural homomorphism from $\boldsymbol{B}$ to $\boldsymbol{C} / \cap\{\operatorname{ker}(f\lceil C) \mid$ $f \in X\}$.

Theorem 2.7 ([6], Theorem 4.3). A finite dualizable algebra that has enough total algebraic operations is strongly dualizable.

Definition 2.8 ([2], p. 73). Let $\boldsymbol{A}$ be an algebra. The enriched partial homclone of $\boldsymbol{A}$ consists of all homomorphisms from $\boldsymbol{B}$ to $\boldsymbol{A}$, for all subalgebras $\boldsymbol{B}$ of $\boldsymbol{A}^{n}$, and all positive integers $n$.

Theorem 2.9 ([2], Brute Force Strong Duality Theorem 3.2.2). Let $\boldsymbol{A}$ be a finite algebra. If some alter ego $\underset{\sim}{\boldsymbol{A}}$ yields a strong duality on $\boldsymbol{A}$, then $\underset{\sim}{\boldsymbol{A}}=\langle A, \mathcal{P}, \tau\rangle$, yields a strong duality on $\boldsymbol{A} \sim$ where $\mathcal{P}$ is the enriched partial hom-clone of $\boldsymbol{A}$ and $\tau$ is the discrete topology on $A$.

Our next result is a special application of the M-shift strong duality Lemma from [2] to the alter ego $\langle A, \mathcal{P}, \tau\rangle$.

Lemma 2.10 ([2], Lemma 3.2.3). Let $\boldsymbol{A}, \mathcal{P}$, and $\tau$ be as in the Theorem 2.9. Let $\mathcal{P}^{\prime} \subseteq \mathcal{P}$ be a generating set of $\mathcal{P}$, that is, every $h \in \mathcal{P}$ is a composition of elements of $\mathcal{P}^{\prime}$ and projections. If $\langle A, \mathcal{P}, \tau\rangle$ yields a strong duality on $\boldsymbol{A}$, then $\left\langle A, \mathcal{P}^{\prime}, \tau\right\rangle$ yields a strong duality on $\boldsymbol{A}$.

\section{A Generating SET FOR DOMAINS OF PARTIAL FUNCTIONS}

In order to show our main result, we want to establish that every affine algebra satisfies the conditions of Definition 2.6, so that we may use Theorem 2.7. The set $X$ appearing in the definition can actually be taken as a set of coordinate projections. Hence to establish a necessary bound on $X$, we need to be able to show that partial compatible functions on $\boldsymbol{A}$ (i.e. homomorphisms from subpowers of $\boldsymbol{A}$ to $\boldsymbol{A}$ ), factor though partial compatible functions of bounded arity. As a first step towards our result, in this section we show that we can generate all possible domains of such functions from a finite set.

The following definitions are from [5]. Given affine algebras $\boldsymbol{A}$ and $\boldsymbol{S}$ and a homomorphism $k: \boldsymbol{A} \rightarrow \boldsymbol{S}$, let $\mathcal{H}_{k}\left(\boldsymbol{A}^{2}, \boldsymbol{S}\right)$ consist of all homomorphisms $f: \boldsymbol{A}^{2} \rightarrow \boldsymbol{S}$ that satisfy $f(x, x)=k(x)$. We set $\bar{k} \in \mathcal{H}_{k}\left(\boldsymbol{A}^{2}, \boldsymbol{S}\right)$ as $\bar{k}(x, y)=k(y)$. In [5, Lemma 5.4], it is shown that $\left\langle\mathcal{H}_{k}\left(\boldsymbol{A}^{2}, \boldsymbol{S}\right) ;+^{\bar{k}}\right\rangle$ is an Abelian group (where $+^{\bar{k}}$ is defined by $f+{ }^{\bar{k}} g=t(f, \bar{k}, g)$ for all $f, g$ in $\left.\mathcal{H}_{k}\left(\boldsymbol{A}^{2}, \boldsymbol{S}\right)\right)$, and that the isomorphism type of $\left\langle\mathcal{H}_{k}\left(\boldsymbol{A}^{2}, \boldsymbol{S}\right) ;+{ }^{\bar{k}}\right\rangle$ does not depend on $k$. We let $\left\langle\mathcal{H}\left(\boldsymbol{A}^{2}, \boldsymbol{S}\right) ;+\right\rangle$ stand for this isomorphism type.

The following lemma, proved in [5, Lemma 5.7], expresses that a (total) homomorphism $f: \boldsymbol{A}^{n} \rightarrow \boldsymbol{S}$ can be factored through a small power of $\boldsymbol{A}$, which does not depend on $n$ but depends only on $\left\langle\mathcal{H}\left(\boldsymbol{A}^{2}, \boldsymbol{S}\right) ;+\right\rangle$.

Lemma 3.1. Let $\boldsymbol{A}, \boldsymbol{S}$ be algebras in a variety of affine algebras. Let $L$ be a positive integer such that $\left\langle\mathcal{H}\left(\boldsymbol{A}^{2}, \boldsymbol{S}\right) ;+\right\rangle$ has a family of generators with $L-1$ elements. Let 
$f: \boldsymbol{A}^{n} \rightarrow \boldsymbol{S}$ be a homomorphism. Then there exists a homomorphism $p: \boldsymbol{A}^{n} \rightarrow \boldsymbol{A}^{L}$ that is a term in $t$, and a homomorphism $q: \boldsymbol{A}^{L} \rightarrow \boldsymbol{S}$, such that $f=q \circ p$.

Corollary 3.2 ([5], Corollary 5.6). Let $\boldsymbol{A}$ and $\boldsymbol{S}$ be affine algebras such that $|A|=$ $p_{1}^{\alpha_{1}} \ldots p_{k}^{\alpha_{k}}$ and $|S|=p_{1}^{\beta_{1}} \ldots p_{k}^{\beta_{k}}$ for distinct primes $p_{i}$. Then $\left|\mathcal{H}\left(\boldsymbol{A}^{2}, \boldsymbol{S}\right)\right|$ divides $p_{1}^{\alpha_{1} \beta_{1}} \ldots p_{k}^{\alpha_{k} \beta_{k}}$, and $\left\langle\mathcal{H}\left(\boldsymbol{A}^{2}, \boldsymbol{S}\right) ;+\right\rangle$ has a generating set of size $\max _{1 \leq i \leq k}\left(\alpha_{i} \beta_{i}\right)$.

Corollary 3.3. Let $\boldsymbol{A}$ be a finite affine algebra. Let $p_{1}^{\alpha_{1}} p_{2}^{\alpha_{2}} \ldots p_{k}^{\alpha_{k}}$ be the prime decomposition of $|A|$. Let $K=1+\max _{1 \leq i \leq k}\left(\alpha_{i}^{3}\right)$. Denote by $\mathcal{F}$ the partial clone over $A$ generated by $t$ and all $\pi_{1}^{K}\left\lceil C\right.$ for $\boldsymbol{C}$ a subalgebra of $\boldsymbol{A}^{K}$. Then $\pi_{1}^{n}\lceil D$ belongs to $\mathcal{F}$ for all positive integers $n$ and all subalgebras $\boldsymbol{D}$ of $\boldsymbol{A}^{n}$.

Proof. Let $n$ be a positive integer and $\boldsymbol{D}$ a completely meet-irreducible subalgebra of $\boldsymbol{A}^{n}$. Set $\boldsymbol{S}=\boldsymbol{A}^{n} / \Theta_{D}$, and denote by $f: \boldsymbol{A}^{n} \rightarrow \boldsymbol{S}$ the canonical projection.

By Lemma 2.2, $\{D\}$ is the underlying set of a (one-element) subalgebra of $\boldsymbol{S}$, moreover $x \in D \Longleftrightarrow f(x)=D$. That is $f^{-1}(\{D\})=D$.

As $\boldsymbol{D}$ is completely meet-irreducible, by Lemma $2.3, \boldsymbol{S}$ is subdirectly irreducible. By Theorem 8.4, $|S|$ divides $p_{1}^{\alpha_{1}^{2}} \ldots p_{k}^{\alpha_{k}^{2}}$. By Corollary 3.2 the group $\mathcal{H}\left(\boldsymbol{A}^{2}, \boldsymbol{S}\right)$ has a generating family with $\max _{1 \leq i \leq k}\left(\alpha_{i}^{3}\right)=K-1$ elements.

Therefore, by Lemma 3.1, there exists a homomorphism $p: \boldsymbol{A}^{n} \rightarrow \boldsymbol{A}^{K}$, which is a term in $t$, and a homomorphism $q: \boldsymbol{A}^{K} \rightarrow \boldsymbol{S}$ such that $q \circ p=f$.

Set $C=q^{-1}(\{D\})=\left\{x \in A^{K} \mid q(x)=D\right\}$. As $\{D\}$ is the underlying set of a subalgebra of $\boldsymbol{S}$ and $q$ is a homomorphism, it follows that $C$ is the underlying set of a subalgebra of $\boldsymbol{A}^{K}$, hence $C$ is a domain of $\mathcal{F}$. Moreover $p$ is a term of $t$, thus it follows from Lemma 2.5(4) that $p^{-1}(C)$ is a domain of $\mathcal{F}$. However $p^{-1}(C)=p^{-1}\left(q^{-1}(\{D\})\right)=f^{-1}(\{D\})=D$, so $D$ is a domain of $\mathcal{F}$.

Let $\boldsymbol{B}$ be an arbitrary subalgebra of $\boldsymbol{A}^{n}$. We can write $B$ as the intersection of finitely many underlying sets of completely subdirectly irreducible subalgebras of $\boldsymbol{A}^{n}$. Since each of these sets is a domain of $\mathcal{F}$, it follows from Lemma 2.5(2) that $B$ is a domain of $\mathcal{F}$. Therefore, by Lemma $2.5(3), \pi_{1}^{n} \uparrow B$ is in $\mathcal{F}$.

\section{Extensions of Partial homomorphisms}

The results of Corollary 3.3 imply that we may generate a partial homomorphism from its extension to a larger domain and a bounded number of partial projections. Thus, the goal of this section is to extend partial homomorphisms of affine algebras (in a finitely generated variety of affine algebras). We will show that if the domain of a partial homomorphism is small enough, then the partial homomorphism has a proper extension (cf. Lemma 4.5). We will first establish this result for modules before generalizing to affine algebras.

Lemma 4.1. Let $\boldsymbol{B}, \boldsymbol{C}$ be submodules of a module $\boldsymbol{A}$. Let $\boldsymbol{E}$ be a module. Let $f: \boldsymbol{B} \rightarrow \boldsymbol{E}$ and $g: \boldsymbol{C} \rightarrow \boldsymbol{E}$ be homomorphisms. If $f\lceil B \cap C=g\lceil B \cap C$, then there exists a homomorphism $h: \boldsymbol{B}+\boldsymbol{C} \rightarrow \boldsymbol{E}$ that is a common extension of $f$ and $g$.

Proof. Let $b, b^{\prime} \in B$ and $c, c^{\prime} \in C$. Assume that $b+c=b^{\prime}+c^{\prime}$. Then $b-b^{\prime}=c^{\prime}-c$ belongs to $B \cap C$, hence

$$
f(b)-f\left(b^{\prime}\right)=f\left(b-b^{\prime}\right)=g\left(b-b^{\prime}\right)=g\left(c^{\prime}-c\right)=g\left(c^{\prime}\right)-g(c) .
$$


Therefore $f(b)+g(c)=f\left(b^{\prime}\right)+g\left(c^{\prime}\right)$. It follows that the map

$$
\begin{aligned}
h: B+C & \rightarrow E \\
b+c & \mapsto f(b)+g(c),
\end{aligned}
$$

is well-defined, and is a homomorphism of modules. Moreover $h(b)=h(b+0)=$ $f(b)+g(0)=f(b)$ for all $b \in B$. Similarly $h$ extends $g$.

Lemma 4.2. Let $\mathcal{V}$ be a locally finite variety of algebras, $\boldsymbol{A} \in \mathcal{V}$, and $n$ a positive integer. If each finitely generated subalgebra of $\boldsymbol{A}$ is generated by $n$ elements, then $\boldsymbol{A}$ is finite.

Proof. Assuming that $\boldsymbol{A}$ is infinite, there is an infinite sequence $\left(x_{i}\right)_{i \in \mathbb{N}}$ of distinct elements of $\boldsymbol{A}$. Let $k=\left|\boldsymbol{F}_{v}(n)\right|$.

Denote by $\boldsymbol{B}$ the subalgebra of $\boldsymbol{A}$ generated by $\left\{x_{0}, x_{1}, x_{2}, \ldots, x_{k}\right\}$. Note that $|B| \geq k+1$, but $\boldsymbol{B}$ is finitely generated, so is generated by $n$ elements, hence $|B| \leq\left|F_{\mathcal{V}}(n)\right|=k$, a contradiction.

Lemma 4.3. Let $\boldsymbol{A}$ be an Abelian group such that $|A|=p_{1}^{\alpha_{1}} \ldots p_{k}^{\alpha_{k}}$, where $p_{1}, \ldots, p_{k}$ are distinct primes. Set $M=1+\alpha_{1}+\cdots+\alpha_{k}$. Let $a_{1}, \ldots, a_{M} \in A$. Then there are integers $u_{1}, \ldots, u_{i-1}$, for some $i$ with $1 \leq i \leq M$, such that $a_{i}=\sum_{j=1}^{i-1} u_{j} a_{j}$.

Proof. Set $A_{0}=\{0\}$. Given $1 \leq i \leq M$, denote by $\boldsymbol{A}_{i}$ the subgroup of $\boldsymbol{A}$ generated by $\left\{a_{1}, \ldots, a_{i}\right\}$. Note that $\boldsymbol{A}_{i}$ is a subgroup of $\boldsymbol{A}_{j}$ for $0 \leq i \leq j \leq M$.

As a maximal chain of subgroups of $\boldsymbol{A}$ has size at most $M$, it follows that there is $1 \leq i \leq M$ such that $A_{i}=A_{i-1}$. Therefore $a_{i} \in A_{i-1}$, so there are integers $u_{1}, \ldots, u_{i-1}$ such that $a_{i}=\sum_{j=1}^{i-1} u_{j} a_{j}$.

Lemma 4.4. Let $\boldsymbol{R}$ be a finite ring and $\mathcal{V}$ the variety of $\boldsymbol{R}$-modules. Let $\boldsymbol{E} \in \mathcal{V}$ be finite. Assume that $|R|=p_{1}^{r_{1}} \ldots p_{k}^{r_{k}}$, and $|E|=p_{1}^{\beta_{1}} \ldots p_{k}^{\beta_{k}}$, where the $p_{i}$ are distinct primes, and that $\boldsymbol{R}$, as an $\boldsymbol{R}$-module, has s proper non-trivial submodules. Set $N=s \times\left(\sum_{i=1}^{k} r_{i} \beta_{i}-1\right)$. Then for all modules $\boldsymbol{B} \leq \boldsymbol{C}$ in $\mathcal{V}$ and all homomorphism $f: \boldsymbol{B} \rightarrow \boldsymbol{E}$, if $\boldsymbol{C} / B$ is not generated by $N$ elements, then $f$ has a proper extension.

Proof. We first find a submodule $\boldsymbol{H}$ of $\boldsymbol{C}$, such that $\boldsymbol{H}$ is not contained in $\boldsymbol{B}$ and $f(H \cap B)=\{0\}$. Lemma 4.1 will then allow us to extend $f$ to $B+H$.

Denote by $\boldsymbol{F}$ the $\boldsymbol{R}$-module freely generated by $\{u\}$, so that $\boldsymbol{F} \cong \boldsymbol{R}$ as $\boldsymbol{R}$ modules.

Let $\boldsymbol{B} \leq \boldsymbol{C}$ in $\mathcal{V}$ and $f: \boldsymbol{B} \rightarrow \boldsymbol{E}$ be a homomorphism. Assume that $\boldsymbol{C} / B$ is not generated by $N$ elements.

First note that if all finitely generated submodules of $C / B$ are generated by $N$ elements, then it follows from Lemma 4.2 that $C / B$ is generated by $N$ elements, which contradicts the assumption. Therefore there is a finitely generated submodule $\boldsymbol{Q}$ of $\boldsymbol{C} / B$, whose minimal number of generators is $k \geq N+1$.

Let $\boldsymbol{P}$ be the submodule of $\boldsymbol{C}$ containing $\boldsymbol{B}$ such that $\boldsymbol{P} / B=\boldsymbol{Q}$. Note that $\left\{x_{1}+B, x_{2}+B, \ldots, x_{k}+B\right\}$ generates $\boldsymbol{Q}$ if and only if $B \cup\left\{x_{1}, \ldots, x_{k}\right\}$ generates $\boldsymbol{P}$. We say that $x_{1}, \ldots, x_{k}$ generate $\boldsymbol{P}$ over $\boldsymbol{B}$.

Given $x \in C$ we denote by $\varphi_{x}: \boldsymbol{F} \rightarrow \boldsymbol{C}$ the unique homomorphism that maps $u$ to $x$. Note that $\varphi_{x}+\varphi_{y}=\varphi_{x+y}$ for all $x, y \in C$.

Pick $x_{1}, \ldots, x_{k} \in P \backslash B$, generating $\boldsymbol{P}$ over $\boldsymbol{B}$, such that $\left(\varphi_{x_{i}}^{-1}(B)\right)_{1 \leq i \leq k}$ is maximal. That is, if $y_{1}, \ldots, y_{k}$ generate $\boldsymbol{P}$ over $\boldsymbol{B}$ and $\varphi_{y_{i}}^{-1}(B) \supseteq \varphi_{x_{i}}^{-1}(B)$ for all 
$1 \leq i \leq k$, then $\varphi_{y_{i}}^{-1}(B)=\varphi_{x_{i}}^{-1}(B)$ for all $1 \leq i \leq k$. The existence of such a sequence follows from the finiteness of Sub $\boldsymbol{F}$.

Set $S_{i}=\varphi_{x_{i}}^{-1}(B)$. That is, $\boldsymbol{S}_{i}$ is the largest submodule of $\boldsymbol{F}$ such that $\varphi_{x_{i}}\left(S_{i}\right) \subseteq$ $B$, for each $1 \leq i \leq k$. Note that $\varphi_{x_{i}}(F) \cap B=\varphi_{x_{i}}\left(S_{i}\right)$.

Assume that $S_{i}=\{0\}$ for some $1 \leq i \leq k$. Then $\varphi_{x_{i}}(F) \cap B=\{0\}$. Let $g: \varphi_{x_{i}}(F) \rightarrow \boldsymbol{E}, x \mapsto 0$. Note that $\operatorname{dom} f \cap \operatorname{dom} g=\{0\}$, hence it follows from Lemma 4.1 that there exist a homomorphism $h: B+\varphi_{x_{i}}(F) \rightarrow \boldsymbol{E}$ that extends both $f$ and $g$. As $x_{i} \notin B$, it follows that $h$ is a proper extension of $f$.

We now assume that $S_{i} \neq\{0\}$ for each $1 \leq i \leq k$.

Assume we have $i$ such that $S_{i}=F$. Hence $u$, the generator of $\boldsymbol{F}$, belongs to $\boldsymbol{S}_{i}$, so $x_{i}=\varphi_{x_{i}}(u) \in \varphi_{x_{i}}\left(S_{i}\right) \subseteq B$, contradicting that $x_{i} \notin B$. Therefore the $\boldsymbol{S}_{i}$ are proper submodules of $\boldsymbol{F}$, for all $1 \leq i \leq n$.

As $k \geq N+1>N$ and the $\boldsymbol{S}_{i}$ are proper nontrivial submodules of $\boldsymbol{F}$, it follows that there is a submodule $\boldsymbol{G}$ of $\boldsymbol{F}$, with $\boldsymbol{G}$ proper and non-trivial, and $I \subseteq$ $\{1, \ldots, k\}$, such that $|I|=\sum_{i=1}^{k} r_{i} \beta_{i}$ and $S_{i}=G$, for all $i \in I$. Set $\psi_{i}=f \circ \varphi_{x_{i}} \uparrow G$, for all $i \in I$.

As $\boldsymbol{G}$ is a proper submodule of $\boldsymbol{F}$, it follows from $\operatorname{Lemma} 8.1$ that $|\operatorname{Hom}(\boldsymbol{G}, \boldsymbol{E})|$ is a proper divisor of $p_{1}^{r_{1} \beta_{1}} \ldots p_{k}^{r_{k} \beta_{k}}$, hence by Lemma 4.3, there is $i \in I$ and a family of integers $\left(u_{j}\right)_{j \in J}$ (where $J=I \backslash\{i\}$ ) such that

$$
\psi_{i}=\sum_{j \in J} u_{j} \psi_{j}
$$

Let $y=x_{i}-\sum_{j \in J} u_{j} x_{j}$. Note that $x_{1}, \ldots, x_{i-1}, y, x_{i+1}, \ldots, x_{k}$ generates $\boldsymbol{P}$ over $\boldsymbol{B}$. Moreover

$$
\varphi_{y}(s)=\varphi_{x_{i}-\sum_{j \in J} u_{j} x_{j}}(s)=\varphi_{x_{i}}(s)-\sum_{j \in J} u_{j} \varphi_{x_{j}}(s) \in B, \quad \text { for all } s \in G
$$

thus $\varphi_{y}^{-1}(B) \supseteq G=S_{i}$. It follows from the maximality of $\left(S_{1}, \ldots, S_{k}\right)$ that $\varphi_{y}^{-1}(B)=S_{i}=G$.

Set $H=\varphi_{y}(F)$. Let $z \in H \cap B$, and take $s \in G$ such that $\varphi_{y}(s)=z$. The following equalities hold

$$
\begin{aligned}
f(z) & =f\left(\varphi_{y}(s)\right) \\
& =f\left(\varphi_{x_{i}}(s)-\sum_{j \in J} u_{j} \varphi_{x_{j}}(s)\right) \\
& =f\left(\varphi_{x_{i}}(s)\right)-\sum_{j \in J} u_{j} f\left(\varphi_{x_{j}}(s)\right) \\
& =\psi_{i}(s)-\sum_{j \in J} u_{j} \psi_{j}(s) \\
& =0
\end{aligned}
$$

Denote by $g: H \rightarrow E$ the constant 0 homomorphism. We have

$$
f \uparrow H \cap B=g \uparrow H \cap B .
$$

Set $D=B+H$. It follows from Lemma 4.1 that $f$ and $g$ have a common extension $h: D \rightarrow E$.

Note that $y=\varphi_{y}(u) \in D$ and $y \notin B$, so $h$ is a proper extension of $f$. 
By [9], every locally finite variety $\mathcal{V}$ of affine algebras is generated by a finite algebra $\boldsymbol{A}$; hence when $\mathcal{V}$ is locally finite we can define the ring associated to $\mathcal{V}$ as the ring associated to $\boldsymbol{A}$.

Lemma 4.5. Let $\mathcal{V}$ be a locally finite variety of affine algebras, and $\boldsymbol{R}$ the ring associated to $\mathcal{V}$. Let $\boldsymbol{E} \in \mathcal{V}$ be finite. Assume that $|R|=p_{1}^{r_{1}} \ldots p_{k}^{r_{k}}$, and $|E|=$ $p_{1}^{\beta_{1}} \ldots p_{k}^{\beta_{k}}$, where the $p_{i}$ are distinct primes, and that $\boldsymbol{R}$, as an $\boldsymbol{R}$-module, has $s$ proper non-trivial submodules. Set $N=s \times\left(\sum_{i=1}^{k} r_{i} \beta_{i}-1\right)$. Then for all algebras $\boldsymbol{B} \leq \boldsymbol{C}$ in $\mathcal{V}$ and all homomorphism $f: \boldsymbol{B} \rightarrow \boldsymbol{E}$, if $\boldsymbol{C} / \Theta_{B}$ is not generated by $N+1$ elements, then $f$ has a proper extension.

Proof. Let $t$ be a Maltsev term of $\mathcal{V}$. Note that we may identify the ring corresponding to $\mathcal{V}$ with the set $R$ of all idempotent binary terms of $\mathcal{V}$ modulo the equational theory of $\mathcal{V}$. Under this correspondence, multiplication is defined by $(\lambda \mu)(x, y)=\lambda(\mu(x, y), y)$, addition is $(\lambda+\mu)(x, y)=t(\lambda(x, y), y, \mu(x, y))$, the zero is $(x, y) \mapsto y$, and the unit is $(x, y) \mapsto x$.

Let $\boldsymbol{A} \in \mathcal{V}$, and for $u \in A$, denote by $+{ }^{u}$ the operation defined over $A$ by $x+{ }^{u} y=t(x, u, y)$. Define an action of $\boldsymbol{R}$ over $A$ by $\lambda x=\lambda(x, u)$ for all $x \in A$, then $\left\langle A ;+^{u}, \boldsymbol{R}\right\rangle$ is a module.

Let $\boldsymbol{B} \leq \boldsymbol{C}$ in $\mathcal{V}$, let $f: \boldsymbol{B} \rightarrow \boldsymbol{E}$ be a homomorphism. Assume that $\boldsymbol{C} / \Theta_{B}$ is not generated by $N+1$ elements. Let $v \in B$, set $w=f(v)$. Note that $\left\langle B ;+^{v}, \boldsymbol{R}\right\rangle$ is a submodule of $\left\langle C ;+^{v}, \boldsymbol{R}\right\rangle$. Moreover, $f:\left\langle B ;+^{v}, \boldsymbol{R}\right\rangle \rightarrow\left\langle E ;+^{w}, \boldsymbol{R}\right\rangle$ is a homomorphism of modules.

Assume that $\left\langle C ;+^{v}, \boldsymbol{R}\right\rangle / B$ is generated by $x_{1}+B, \ldots, x_{N}+B$. It follows that $C / \Theta_{B}$ is generated by $B=v+B, x_{1}+B, \ldots, x_{N}+B$, and so is generated by $N+1$ elements; a contradiction. Therefore $\left\langle C ;+^{v}, \boldsymbol{R}\right\rangle / B$ is not generated by $N$ elements. It follows from Lemma 4.4 that as a module homomorphism, $f$ has a proper extension $g$. We claim that $g$ is also an extension of the $\mathcal{V}$-homomorphism $f$.

Denote by $D$ the domain of $g$. Note that $\boldsymbol{C}$ is a reduct of the module structure $\left\langle C ;+^{v}, \boldsymbol{R}\right\rangle$ extended with the constants of $\mathcal{V}$. Moreover, all constants of $\mathcal{V}$ are in $B \subseteq D$, and $D$ is the universe of a submodule of $\left\langle C ;+^{v}, \boldsymbol{R}\right\rangle$. It follows that $\boldsymbol{D}$ is a subalgebra of $\boldsymbol{C}$, and that $g: \boldsymbol{D} \rightarrow \boldsymbol{E}$ is a homomorphism. Hence $f$ has a proper extension.

\section{FACTORING PARTIAL HOMOMORPHISMS}

The main goal of this section is to factorize a partial homomorphism $f: \boldsymbol{C} \rightarrow \boldsymbol{E}$ (where $\boldsymbol{C} \leq \boldsymbol{A}^{n}$ ) through a smaller power $\boldsymbol{D} \leq \boldsymbol{A}^{N}$, where $N$ only depends on $\boldsymbol{A}$ and $\boldsymbol{E}$. This will allow us to use Theorem 2.7 and to prove our main result.

First note that affine algebras have the congruence extension property. To be more precise we give the following description of extensions of congruences.

Lemma 5.1. Let $\boldsymbol{A}$ be a subalgebra of an affine algebra $\boldsymbol{B}$. Let $\alpha$ be a congruence of $\boldsymbol{A}$. Then there exists a smallest extension of $\alpha$ to $\boldsymbol{B}$. It is the unique congruence $\beta$ of $\boldsymbol{B}$ satisfying the following conditions.

(1) For all $(x, y) \in \beta$, if $y \in A$, then $x \in A$.

(2) $\beta \cap A^{2}=\alpha$.

Proof. Pick $0 \in A$ and set $x+y=t(x, 0, y)$. It follows that $A$ is stable for + . Also note that $x-y=t(x, y, 0)$ and $t(x, y, z)=x-y+z$. 
Let $\left(x, x^{\prime}\right) \in \alpha,\left(y, y^{\prime}\right) \in \alpha$. As $\alpha$ is compatible with $t$, it follows that

$$
\left(x+y, x^{\prime}+y^{\prime}\right)=\left(t(x, 0, y), t\left(x^{\prime}, 0, y^{\prime}\right)\right) \in \alpha .
$$

Therefore $\alpha$ is compatible with + and - .

Define $\beta=\left\{(x, y) \in B^{2} \mid(\exists a \in B)((x-a, y-a) \in \alpha)\right\}$. We will leave it to the reader to check that $\beta$ is a congruence that satisfies conditions (1) and (2).

Let $\gamma$ be a congruence of $\boldsymbol{B}$ containing $\alpha$. Let $x, y, a \in B$ such that $(x-a, y-a) \in$ $\alpha$, so $(x-a, y-a) \in \gamma$, hence $(x, y)=(x-a+a, y-a+a) \in \gamma$. Therefore $\gamma$ contains $\beta$, hence $\beta$ is the smallest extension of $\alpha$ to $\boldsymbol{B}$.

Let $\gamma$ be a congruence satisfying (1) and (2). First note that $\gamma$ contains $\alpha$, so $\gamma$ contains $\beta$. Conversely, let $(x, y) \in \gamma$. Note that $(x-y, 0)=(x-y, y-y) \in \gamma$ and $0 \in A$, so $x-y \in A$, and so $(x-y, y-y) \in \gamma \cap A^{2}=\alpha$, that is $(x, y) \in \beta$. Therefore $\gamma=\beta$.

Lemma 5.2. Let $\boldsymbol{A} \leq \boldsymbol{B}$ be finite affine algebras. Let $\alpha$ be a congruence of $\boldsymbol{A}$. Let $\beta$ be the minimal extension of $\alpha$ to $\boldsymbol{B}$. Then $|B / \beta|=\left|B / \Theta_{A}\right| \times|A / \alpha|$.

Proof. First note that $\Theta_{A} \supseteq \alpha$, hence $\Theta_{A} \supseteq \beta$. Also note that $\boldsymbol{A} / \beta$ is a subalgebra of $\boldsymbol{B} / \beta$.

We first show that $\Theta_{A} / \beta=\Theta_{A / \beta}$. Let $(x / \beta, y / \beta) \in \Theta_{A / \beta}$. There is $u \in A / \beta$ such that $y / \beta-x / \beta+u \in A / \beta$, hence there is $c \in A$ such that $(y-x+c) / \beta \in A / \beta$. That is $(y-x+c) / \beta=a / \beta$ for some $a \in A$. It follows from Lemma 5.1(1) that $(y-x+c) \in A$. Therefore $(x, y) \in \Theta_{A}$, hence $(x / \beta, y / \beta) \in \Theta_{A} / \beta$.

Conversely, let $(x / \beta, y / \beta) \in \Theta_{A} / \beta$. That is $(x, y) \in \Theta_{A}$. There is $c \in A$ such that $x-y+c \in A$, so $x / \beta-y / \beta+c / \beta \in A / \beta$. As $c / \beta \in A / \beta$, it follows that $(x / \beta, y / \beta) \in \Theta_{A / \beta}$. This proves that $\Theta_{A / \beta}=\Theta_{A} / \beta$.

Note that $\boldsymbol{B} / \Theta_{A} \cong(\boldsymbol{B} / \beta) /\left(\Theta_{A} / \beta\right)=(\boldsymbol{B} / \beta) /\left(\Theta_{A / \beta}\right)$. Lemma 8.5 implies that $\left|B / \Theta_{A}\right|=|B / \beta| /|A / \beta|$. Now $\beta \cap A^{2}=\alpha$, so $\boldsymbol{A} / \beta \cong \boldsymbol{A} / \alpha$, and therefore $|B / \beta|=$ $\left|B / \Theta_{A}\right| \times|A / \beta|$.

Theorem 5.3. Let $\boldsymbol{A}, \boldsymbol{E}$ be finite affine algebras of the same type, and $\mathcal{V}$ be the variety generated by $\{\boldsymbol{A}, \boldsymbol{E}\}$. Assume that $|A|=p_{1}^{\alpha_{1}} \ldots p_{k}^{\alpha_{k}}$, and $|E|=p_{1}^{e_{1}} \ldots p_{k}^{e_{k}}$, for distinct primes $p_{1}, \ldots, p_{k}$. For $N$ as in Lemma 4.5 , let $\left|F_{\mathcal{V}}(N+1)\right|=p_{1}^{u_{1}} \ldots p_{k}^{u_{k}}$, and set

$$
\ell=1+\max _{1 \leq i \leq k}\left(\alpha_{i}\left(u_{i}+e_{i}\right)\right)
$$

Then for every positive integer n, every subalgebra $\boldsymbol{C}$ of $\boldsymbol{A}^{n}$, and every homomorphism $h: \boldsymbol{C} \rightarrow \boldsymbol{E}$, there exists homomorphisms $p: \boldsymbol{A}^{n} \rightarrow \boldsymbol{A}^{\ell}$ and $k: p(\boldsymbol{C}) \rightarrow \boldsymbol{E}$ such that $k \circ p\lceil C=h$. Moreover, we can choose $p$ to be a term in $t$, the Maltsev term of $\mathcal{V}$.

Proof. Note that most of the homomorphisms and commuting relations used in this proof are illustrated in Figure 1. Set $P=\left|F_{\mathcal{V}}(N+1)\right| \times|E|=p_{1}^{u_{1}+e_{1}} \ldots p_{k}^{u_{k}+e_{k}}$.

Let $n$ be a positive integer, $\boldsymbol{C} \in \operatorname{Sub}\left(\boldsymbol{A}^{n}\right)$, and $h: \boldsymbol{C} \rightarrow \boldsymbol{E}$ a homomorphism. Let $\boldsymbol{D}$ be a subalgebra of $\boldsymbol{A}^{n}$, such that $h^{\prime}: \boldsymbol{D} \rightarrow \boldsymbol{E}$ is a maximal extension of $h$. Denote by $\eta_{1}: \boldsymbol{C} \rightarrow \boldsymbol{D}$ the inclusion homomorphism. As $h^{\prime}$ extends $h$, we have

$$
h^{\prime} \circ \eta_{1}=h \text {. }
$$

It follows from Lemma 4.5 that $\boldsymbol{A}^{n} / \Theta_{D}$ is generated by $N+1$ elements, hence $\left|\boldsymbol{A}^{n} / \Theta_{D}\right|$ divides $\left|F_{\mathcal{V}}(N+1)\right|$. Let $\varepsilon_{1}: \boldsymbol{D} \rightarrow \boldsymbol{A}^{n}$ be the inclusion homomorphism. 
Denote by $\alpha$ the kernel of $h^{\prime}$ and by $\beta$ the minimal extension of $\alpha$ to $\boldsymbol{A}^{n}$. Set $\boldsymbol{R}=\boldsymbol{D} / \alpha$ and $\boldsymbol{S}=\boldsymbol{A}^{n} / \beta$. Let $\varepsilon_{2}: \boldsymbol{R} \rightarrow \boldsymbol{S}$ be the canonical embedding. Let $\pi: \boldsymbol{D} \rightarrow \boldsymbol{R}$ and $\pi^{\prime}: \boldsymbol{A}^{n} \rightarrow \boldsymbol{S}$ be the canonical projections. Note that

$$
\pi^{\prime} \circ \varepsilon_{1}=\varepsilon_{2} \circ \pi .
$$

As $h^{\prime}$ factors through $\pi$, there is an embedding $\sigma: \boldsymbol{R} \rightarrow \boldsymbol{E}$ such that

$$
h^{\prime}=\sigma \circ \pi \text {. }
$$

Hence $|\boldsymbol{R}|=|\boldsymbol{D} / \alpha|$ divides $|\boldsymbol{E}|$. It follows from Lemma 5.2 that

$$
|\boldsymbol{S}|=\left|\boldsymbol{A}^{n} / \beta\right|=\left|\boldsymbol{A}^{n} / \Theta_{D}\right| \times|\boldsymbol{D} / \alpha| \text { divides } P,
$$

hence, by Corollary 3.2 , the algebra $\left\langle\mathcal{H}\left(\boldsymbol{A}^{2}, \boldsymbol{S}\right) ;+\right\rangle$ has a generating family with $\max _{1 \leq i \leq k}\left(\alpha_{i}\left(e_{i}+u_{i}\right)\right)=\ell-1$ elements.

From Lemma 3.1, we have a homomorphism $p: \boldsymbol{A}^{n} \rightarrow \boldsymbol{A}^{\ell}$, which is a term in $t$, and $q: \boldsymbol{A}^{\ell} \rightarrow \boldsymbol{S}$ such that

$$
q \circ p=\pi^{\prime} .
$$

As $\boldsymbol{D}$ is a subalgebra of $\boldsymbol{A}^{n}$, it follows that $p(\boldsymbol{D})$ is a subalgebra of $\boldsymbol{A}^{\ell}$. Denote by $\varepsilon_{3}: p(\boldsymbol{D}) \rightarrow \boldsymbol{A}^{\ell}$ the canonical embedding. Note that

$$
\varepsilon_{3} \circ p\left\lceil\boldsymbol{D}=p \circ \varepsilon_{1} .\right.
$$

Similarly we denote by $\eta_{2}: p(\boldsymbol{C}) \rightarrow p(\boldsymbol{D})$ the inclusion homomorphism, so

$$
\eta_{2} \circ p\left\lceil\boldsymbol{C}=p\left\lceil\boldsymbol{D} \circ \eta_{1} .\right.\right.
$$

The following equalities are direct consequences of (5.2), (5.4), and (5.5)

$$
q \circ \varepsilon_{3} \circ p \nmid \boldsymbol{D}=q \circ p \circ \varepsilon_{1}=\pi^{\prime} \circ \varepsilon_{1}=\varepsilon_{2} \circ \pi .
$$

So $q\left(\varepsilon_{3}(p(D))\right)=\varepsilon_{2}(\pi(D))=\varepsilon_{2}(R)$. However, $\varepsilon_{2}$ is an embedding, so $q\left(\varepsilon_{3}(p(D))\right)$ corresponds to a subalgebra of $R$; hence, there is a homomorphism $u: p(\boldsymbol{D}) \rightarrow \boldsymbol{R}$ such that

$$
q \circ \varepsilon_{3}=\varepsilon_{2} \circ u
$$

It follows from (5.8) and (5.7) that $\varepsilon_{2} \circ u \circ p\left\lceil D=q \circ \varepsilon_{3} \circ p\left\lceil\boldsymbol{D}=\varepsilon_{2} \circ \pi\right.\right.$. As $\varepsilon_{2}$ is an embedding it follows that

$$
u \circ p \uparrow \boldsymbol{D}=\pi .
$$

The following equalities hold

$$
\begin{aligned}
\sigma \circ u \circ \eta_{2} \circ p\lceil\boldsymbol{C} & =\sigma \circ u \circ p\left\lceil\boldsymbol{D} \circ \eta_{1}\right. & & \text { by }(5.6) . \\
& =\sigma \circ \pi \circ \eta_{1} & & \text { by }(5.9) . \\
& =h^{\prime} \circ \eta_{1} & & \text { by }(5.3) . \\
& =h & & \text { by }(5.1) .
\end{aligned}
$$

Therefore, denoting $k=\sigma \circ u \circ \eta_{2}: p(\boldsymbol{C}) \rightarrow \boldsymbol{E}$, we have $k \circ p\lceil\boldsymbol{C}=h$.

Corollary 5.4. Let $\boldsymbol{A}$ be a finite affine algebra, and $p_{1}^{\alpha_{1}} \ldots p_{k}^{\alpha_{k}}$ the prime decomposition of $|A|$. The enriched partial hom-clone of $\boldsymbol{A}$ is finitely generated by partial operations of arity at most

$$
1+\max _{1 \leq i \leq k}\left((Q+1) \alpha_{i}^{3}+2 \alpha_{i}^{2}\right)
$$




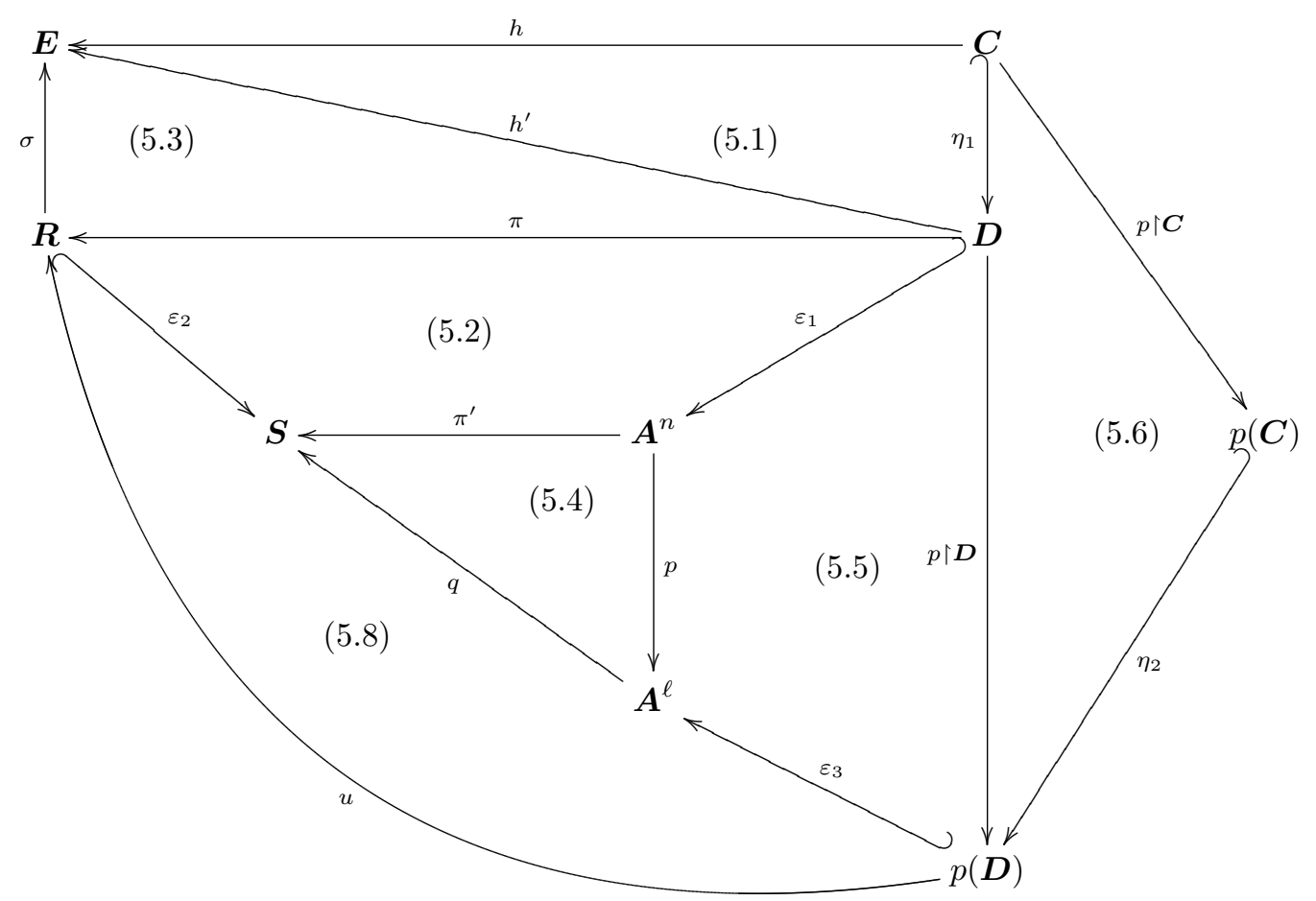

FiguRE 1. Homomorphisms factor through sub-power of small dimension.

where $Q=p_{1}^{\alpha_{1}^{4}} \ldots p_{k}^{\alpha_{k}^{4}} \times\left(\sum_{i=1}^{k} \alpha_{i}^{3}-1\right)$.

Proof. We may assume that $\boldsymbol{A}$ is non-trivial. Let $\ell$ be the bound in Theorem 5.3 for the special case $\boldsymbol{E}=\boldsymbol{A}$. That is,

$$
\ell=1+\max _{1 \leq i \leq k}\left(\alpha_{i}\left(u_{i}+\alpha_{i}\right)\right)
$$

where $\left|F_{\mathcal{V}}(N+1)\right|=p_{1}^{u_{1}} \ldots p_{k}^{u_{k}}, N$ is as in Lemma 4.5 and $\mathcal{V}$ is the variety generated by $\boldsymbol{A}$. Moreover, let $K=1+\max _{1 \leq i \leq k}\left(\alpha_{i}^{3}\right)$ (i.e. $K$ is the bound from Corollary 3.3).

Given integers $1 \leq i \leq n$, we denote by $\pi_{i}^{n}: \boldsymbol{A}^{n} \rightarrow \boldsymbol{A}$ the canonical projection on the $i$-th coordinate. Set $X=\left\{f: \boldsymbol{D} \rightarrow \boldsymbol{A} \mid \boldsymbol{D}\right.$ is a subalgebra of $\left.\boldsymbol{A}^{\ell}\right\}$ and $Y=\left\{\pi_{1}^{K}\left\lceil C \mid \boldsymbol{C}\right.\right.$ is a subalgebra of $\left.\boldsymbol{A}^{K}\right\}$.

Denote by $\mathcal{F}$ the partial clone generated by $\{t\} \cup X \cup Y$.

Let $n$ be a positive integer, $\boldsymbol{C}$ a subalgebra of $\boldsymbol{A}^{n}$, and $h: \boldsymbol{C} \rightarrow \boldsymbol{A}$ a homomorphism. By Theorem 5.3, there is a term $p: \boldsymbol{A}^{n} \rightarrow \boldsymbol{A}^{\ell}$ in $t$, and a homomorphism $k: p(\boldsymbol{C}) \rightarrow \boldsymbol{A}$ such that $h=k \circ p\lceil C$.

Note that $k \in \mathcal{F}$, as it is a partial homomorphism of arity $\ell$, so $k \circ p$ belongs to $\mathcal{F}$. Moreover, by Corollary 3.3, $\pi_{1}^{n} \uparrow C$ belongs to $\mathcal{F}$, therefore, by Lemma $2.5(3)$, $h=k \circ p\lceil C$ belongs to $\mathcal{F}$.

Therefore $\mathcal{F}$ is the set of all partial operations on $A$, compatible with $\boldsymbol{A}$. Moreover, $\mathcal{F}$ is, by construction, finitely generated by partial operations of arity at most $\max \{3, \ell, K\}$. Clearly, $K$ and 3 are smaller than the bound from the statement of the corollary, as we assumed that $\boldsymbol{A}$ is non-trivial. 
It remains to bound the quantity $\ell$. Consider the ring $\boldsymbol{R}$ associated to $\mathcal{V}$. By Lemma 2.4 the cardinality of $\boldsymbol{R}$ divides $p_{1}^{\alpha_{1}^{2}} \ldots p_{k}^{\alpha_{k}^{2}}$. It follows from Lemma 8.1 that $\boldsymbol{R}$, as a $\boldsymbol{R}$-module, has at most $p_{1}^{\alpha_{1}^{4}} \ldots p_{k}^{\alpha_{k}^{4}}$ submodules, and thus $Q \geq N$.

From Lemma 8.6 we have

$$
\left|F_{\mathcal{V}}(N+1)\right| \text { divides } p_{1}^{(N+1) \alpha_{1}^{2}+\alpha_{1}} \ldots p_{k}^{(N+1) \alpha_{k}^{2}+\alpha_{k}} .
$$

Therefore

$$
\left.\ell \leq 1+\max _{1 \leq i \leq k}\left(\alpha_{i}\left((N+1) \alpha_{i}^{2}+\alpha_{i}+\alpha_{i}\right)\right) \leq 1+\max _{1 \leq i \leq k}\left((Q+1) \alpha_{i}^{3}+2 \alpha_{i}^{2}\right)\right) .
$$

The results follows.

We remark in passing that our corollary provides an additional proof that every finite affine algebra $\boldsymbol{A}$ is dualizable (even though it is unnecessarily complicated compared to the arguments in [5]). Dualizability of $\boldsymbol{A}$ follows as by the Duality Compactness Theorem [10, 11], it suffices to show that the enriched partial homclone of $\boldsymbol{A}$ is finitely generated. Our arguments are however not independent, as we rely on Lemmas $2.2,2.3$, and 3.1 from [5].

We are now ready to prove our final result about the strong dualizability of affine algebras.

Lemma 5.5. All finite affine algebras have enough total algebraic operations.

Proof. Let $\boldsymbol{A}$ be an affine algebra, let $\ell$ be as in Theorem 5.3 for $\boldsymbol{A}=\boldsymbol{E}$. We consider $\varphi: \omega \rightarrow \omega$ the constant map equal to $\ell$.

Let $n$ be a positive integer, and let $\boldsymbol{B} \leq \boldsymbol{C}$ be subalgebras of $\boldsymbol{A}^{n}$. Denote by $\iota: \boldsymbol{B} \rightarrow \boldsymbol{C}$ the inclusion homomorphism. Let $h: \boldsymbol{B} \rightarrow \boldsymbol{A}$, let $h^{\prime}: \boldsymbol{C} \rightarrow \boldsymbol{A}$ be an extension of $h$, that is $h^{\prime} \circ \iota=h$.

By Theorem 5.3 there exist a homomorphism $p: \boldsymbol{A}^{n} \rightarrow \boldsymbol{A}^{\ell}$ and a homomorphism $k: p(\boldsymbol{C}) \rightarrow \boldsymbol{A}$ such that $h^{\prime}=k \circ p\left\lceil C\right.$. We obtain $k \circ p\left\lceil C \circ \iota=h^{\prime} \circ \iota=h\right.$.

For each $1 \leq i \leq \ell$, denote by $\pi_{i}: \boldsymbol{A}^{\ell} \rightarrow \boldsymbol{A}$ the canonical projection. Set $X=\left\{\pi_{i} \circ p \mid 1 \leq i \leq \ell\right\}$, hence $X \subseteq \operatorname{Hom}\left(\boldsymbol{A}^{n}, \boldsymbol{A}\right)$, moreover $|X| \leq \ell=\varphi(|B|)$. Note that

$$
\bigcap\left\{\operatorname{ker}(f\lceil C) \mid f \in X\}=\bigcap\left\{\operatorname{ker}\left(\pi_{i} \circ p\lceil C) \mid 1 \leq i \leq \ell\right\}=\operatorname{ker}(p\lceil C) .\right.\right.
$$

Denote by $\eta: \boldsymbol{C} / \operatorname{ker}(p\lceil C) \rightarrow p(\boldsymbol{C})$ the isomorphism induced by $p$, and by $\alpha: \boldsymbol{B} \rightarrow$ $\boldsymbol{C} / \operatorname{ker}(p\lceil C)$ the natural homomorphism. We obtain $\eta \circ \alpha=p\lceil B$. Set $q=k \circ \eta$. We have $q \circ \alpha=k \circ \eta \circ \alpha=k \circ p \uparrow B=k \circ p \uparrow C \circ \iota=h$. Therefore $\boldsymbol{A}$ has enough total algebraic operations (cf. Definition 2.6)

Theorem 5.6. All finite affine algebras are strongly dualizable.

Proof. Let $\boldsymbol{A}$ be a finite affine algebra. By Lemma 5.5, $\boldsymbol{A}$ has enough total algebraic operations, moreover $\boldsymbol{A}$ is dualizable ([5], see also the remark after Corollary 5.4). By Theorem 2.7, $\boldsymbol{A}$ is strongly dualizable.

Our main Theorem 1.1 now follows from the well known fact that any strongly dualizable algebra is fully dualizable (see for example [2, Theorem 3.2.4]). In our final result, we provide an explicit bound on the partial functions in the strongly dualizing alter ego. 
Theorem 5.7. Let $\boldsymbol{A}$ be a finite affine algebra with $|A|=p_{1}^{\alpha_{1}} \ldots p_{k}^{\alpha_{k}}$. Then $\boldsymbol{A}$ is strongly dualized by $\langle A ; \mathcal{P}, \tau\rangle$, where $\tau$ is the discrete topology on $A$ and $\mathcal{P}$ is the set of all compatible partial operations on $\boldsymbol{A}$ of arity at most

$$
1+\max _{1 \leq i \leq k}\left((Q+1) \alpha_{i}^{3}+2 \alpha_{i}^{2}\right),
$$

where $Q=p_{1}^{\alpha_{1}^{4}} \ldots p_{k}^{\alpha_{k}^{4}} \times\left(\sum_{i=1}^{k} \alpha_{i}^{3}-1\right)$.

Proof. As $\boldsymbol{A}$ is strongly dualizable, it is in particular strongly dualized by the strong brute force alter ego $\langle A ; \mathcal{P}, \tau\rangle$ of Theorem 2.9. Moreover, by Lemma 2.10 we may replace $\mathcal{P}$ with a generating set, and by Corollary $5.4, \mathcal{P}$ is finitely generated by partial operations whose arity is limited by the stated bound. The result follows.

\section{EXAMPLE}

We apply our results to an algebra whose examination was crucial in developing the proofs of the previous sections.

Let $\mathcal{F}_{2}$ be the 2 -element field. Consider the 8-element ring $\boldsymbol{R}=\mathcal{F}_{2}[x, y] / I$, where $I$ is the ideal generated by $\left\{x^{2}, y^{2}, x y, y x\right\}$. Let $\boldsymbol{A}$ be the module that is obtained by considering $\boldsymbol{R}$ as a module over itself.

By [5], $\boldsymbol{A}$ is dualizable by an alter ego that includes all compatible relations of size 28. By our main result, $\boldsymbol{A}$ is strongly dualizable. A direct application of Theorem 5.7 will result in a very large bound of $702 \cdot 2^{81}+46$ on the arities of the partial operations in the alter ego.

By adapting the results of the previous sections to this specific example, we can show that a lower bound suffices. As $\boldsymbol{A}$ generates a variety $\mathcal{V}$ of $\boldsymbol{R}$-modules, we know the cardinality of the corresponding ring directly. It also means that we can use Lemma 4.4 instead of Lemma 4.5 in our further arguments, which reduces the " $Q+1$ "-factor in Theorem 5.7 by 1 . Also, the estimate $Q$ can be replaced by exact calculations. Instead of the first factor, we can use the number of nontrivial proper submodules of $\boldsymbol{A}$, which is easily seen to be 4 . The second factor of $Q$ can be replaced by 1 more than the exponent of 2 in the maximal number of homomorphisms from a proper $\boldsymbol{A}$-submodule $\boldsymbol{G}$ to $\boldsymbol{A}$. This number can be shown to be 5 , meaning that $Q+1$ can be replaced with 20 . It then follows that we may obtain a strong duality by using an alter ego with "only" the compatible partial operations of arity 559 .

\section{Problems}

We close with several problems motivated by our results.

Problem 1. Which Abelian algebras that do not generate congruence-modular varieties are dualizable? Which are fully and strongly dualizable?

Problem 2. Are nilpotent dualizable algebras (from congruence-modular varieties) always fully dualizable? Are they strongly dualizable?

We remark that in many well-behaved classes of algebras, dualizability, full dualizability and strong dualizability coincide. Among nilpotent algebras, the results of [1] show that in the subclass of supernilpotent algebras, all non-Abelian algebras are non-dualizable (and by [1] supernilpotence may be replaced by a slightly weaker condition). 
Problem 3. Can the arity bound in our main theorem be improved upon?

We conjecture that a bound of the form $\left(\log _{2}|A|\right)^{n}$ suffices, for some fixed integer $n$.

Problem 4. Which affine algebras are strongly dualized by some alter ego that is a total structure?

\section{Appendix: Counting homomorphisms And Algebras}

Lemma 8.1. Let $\boldsymbol{E}, \boldsymbol{F}$ be Abelian groups. Assume that $|E|=p_{1}^{\alpha_{1}} \ldots p_{k}^{\alpha_{k}}$ and $|F|=p_{1}^{\beta_{1}} \ldots p_{k}^{\beta_{k}}$, where the $p_{i}$ are distinct primes. Then the following statements hold.

(1) The number of subgroups of $\boldsymbol{E}$ is at most $p_{1}^{\alpha_{1}^{2}} \ldots p_{k}^{\alpha_{k}^{2}}$.

(2) $|\operatorname{Hom}(\boldsymbol{F}, \boldsymbol{E})|$ divides $p_{1}^{\alpha_{1} \beta_{1}} \ldots p_{k}^{\alpha_{k} \beta_{k}}$.

Proof. Note that Abelian subgroups of $\boldsymbol{E}$ are determined by their $p_{i}$-Sylow subgroups. Denote by $\boldsymbol{E}_{i}$ the $p_{i}$-Sylow subgroup of $\boldsymbol{E}$, such that $\left|E_{i}\right|=p_{i}^{\alpha_{i}}$. Each subgroup of $\boldsymbol{E}_{i}$ has a family of generators with $\alpha_{i}$ elements. Therefore $\boldsymbol{E}_{i}$ has at most $p_{i}^{\alpha_{i}^{2}}$ subgroups. It follows that (1) holds.

We refer to $[5$, Lemma 4.1$]$ for $(2)$.

Lemma 8.2. Let $\boldsymbol{E}, \boldsymbol{F}$ be affine algebras. Assume that $|E|=p_{1}^{\alpha_{1}} \ldots p_{k}^{\alpha_{k}}$ and $|F|=p_{1}^{\beta_{1}} \ldots p_{k}^{\beta_{k}}$, where the $p_{i}$ are distinct primes. Then the following statements hold.

(1) The number of subalgebras of $\boldsymbol{E}$ is at most $p_{1}^{1+\alpha_{1}^{2}} \ldots p_{k}^{1+\alpha_{k}^{2}}$.

(2) $|\operatorname{Hom}(\boldsymbol{F}, \boldsymbol{E})|$ divides $p_{1}^{\left(\alpha_{1}+1\right) \beta_{1}} \ldots p_{k}^{\left(\alpha_{k}+1\right) \beta_{k}}$.

Proof. For each $c \in E, x+{ }_{c} y=t(x, c, y)$ induces an Abelian group structure on $E$. There are $|E|$ such structures. Let $\boldsymbol{A}$ be a subalgebra of $\boldsymbol{E}$, then for $c \in A,\left\langle A ;+_{c}\right\rangle$ is a subgroup of $\left\langle E ;+_{c}\right\rangle$. With Lemma 8.1, the number of subalgebras of $\boldsymbol{E}$ is at $\operatorname{most}|E| \times p_{1}^{\alpha_{1}^{2}} \ldots p_{k}^{\alpha_{k}^{2}}=p_{1}^{1+\alpha_{1}^{2}} \ldots p_{k}^{1+\alpha_{k}^{2}}$.

We refer to [5, Lemma 4.1] for (2).

Lemma 8.3 ([5], Lemma 4.2). Let $\boldsymbol{E}$ be an affine algebra such that $|E|=p_{1}^{\alpha_{1}} \ldots p_{k}^{\alpha_{k}}$, where the $p_{i}$ are distinct primes, and let $M=1+\max _{1 \leq i \leq k}\left(\alpha_{i}\right)$. Then $\boldsymbol{E}$ has a generating set with $M$ elements.

The following theorem is a particular case of Kearnes' result in [7], also see [5, Corollary 4.4].

Theorem 8.4. Let $\boldsymbol{A}$ be finite affine algebra. Let $p_{1}^{\alpha_{1}} \ldots p_{k}^{\alpha_{k}}$ be the prime decomposition of $|A|$. Let $\boldsymbol{S}$ be a subdirectly irreducible algebra in $\operatorname{Var} \boldsymbol{A}$. Then $|S|$ divides $p_{1}^{\alpha_{1}^{2}} \ldots p_{k}^{\alpha_{k}^{2}}$.

Lemma 8.5. Let $\boldsymbol{A} \leq \boldsymbol{B}$ be affine algebras, then $\left|\boldsymbol{B} / \Theta_{A}\right|=|\boldsymbol{B}| /|\boldsymbol{A}|$.

Proof. Affine algebras are well known to have congruence classes of equal cardinality (see for example [3, Corollary 7.5]). As $A$ is a congruence class of $\Theta_{A}$, the result follows. 
Lemma 8.6. Let $\boldsymbol{A}$ be an affine algebra, with $|A|=p_{1}^{\alpha_{1}} \ldots p_{k}^{\alpha_{k}}$ where the $p_{i}$ are distinct primes. Set $\mathcal{V}=\operatorname{Var} \boldsymbol{A}$. Let $L \in \mathbb{N}$. Then

$$
\left|\boldsymbol{F}_{\mathcal{V}}(L)\right| \text { divides } p_{1}^{L \alpha_{1}^{2}+\alpha_{1}} \ldots p_{k}^{L \alpha_{k}^{2}+\alpha_{k}} \text {. }
$$

Proof. We may identify $\boldsymbol{F}_{\mathcal{V}}(L)$ with the term clone $\operatorname{Clo}_{L}(\boldsymbol{A})$. Each $s: A^{L} \rightarrow$ $A$ in $\operatorname{Clo}_{L}(\boldsymbol{A})$ is compatible with $t$, moreover $\mathrm{Clo}_{L}(\boldsymbol{A})$ is stable under $t$, therefore $\left\langle\operatorname{Clo}_{L}(\boldsymbol{A}) ; t\right\rangle$ is a subalgebra of $\left\langle\operatorname{Hom}\left(\langle A, t\rangle^{L},\langle A, t\rangle\right) ; t\right\rangle$. Hence $\left|\boldsymbol{F}_{\mathcal{V}}(L)\right|=$ $\left|\operatorname{Clo}_{L}(\boldsymbol{A})\right|$ divides $\left|\operatorname{Hom}\left((A, t)^{L},(A, t)\right)\right|$ which itself divides $p_{1}^{L \alpha_{1}^{2}+\alpha_{1}} \ldots p_{k}^{L \alpha_{k}^{2}+\alpha_{k}}$ by Lemma 8.2(2).

\section{ACKNOWLEDGMENTS}

We would like to thank the referee for his or her helpful comments, and, in particular, for suggesting improvements to Lemma 2.4, Corollary 5.4, and Lemma 8.6.

The first author has received funding from the European Union Seventh Framework Programme (FP7/2007-2013) under grant agreement no. PCOFUND-GA2009-246542 and from the Foundation for Science and Technology of Portugal under PCOFUND-GA-2009-246542, SFRH/BCC/52684/2014, and through the CAUL / CEMAT project.

The second author was supported by CONICYT, Proyectos Regulares FONDECYT no. 1150595, by project number P27600 of the Austrian Science Fund (FWF), and by FCT under the supplementary support scheme for PCOFUND-GA-2009246542 .

The third author was supported by National Funding from FCT - Fundação para a Ciência e a Tecnologia, under the project CEMAT-Ciências (UID/Multi/4621/2013)

\section{REFERENCES}

[1] W. Bentz and P. Mayr, Supernilpotence prevents dualizability, Journal of the Australian Mathematical Society 96, 1-24, 2014.

[2] D. M. Clark and B. A. Davey, Natural dualities for the working algebraist. Cambridge Studies in Advanced Mathematics, 57. Cambridge University Press, Cambridge, 1998.

[3] R. Freese and R. McKenzie, Commutator theory for congruence modular varieties. London Mathematical Society Lecture Note Series, 125. Cambridge University Press, Cambridge, 1987.

[4] B. A. Davey, R. Quackenbush, Bookkeeping duality for paraprimal algebras. Contributions to general algebra, 9 (Linz, 1994), 19-26, Hölder-Pichler-Tempsky, Vienna, 1995.

[5] P. Gillibert, Abelian algebras are dualizable, preprint, http://arxiv.org/abs/1503.02651v1

[6] W. A. Lampe, G. F. McNulty and R. Willard, Full duality among graph algebras and flat graph algebras, Algebra Universalis 45, 311-334, 2001.

[7] K. A. Kearnes, Residual bounds for varieties of modules, Algebra Universalis 28, 448-452, 1991.

[8] K. Kearnes and Á. Szendrei, Dualizable algebras with parallelogram terms, Algebra Universalis 76(4), 157-168, 2016.

[9] K. A. Kearnes and R. Willard, Finiteness properties of locally finite abelian varieties, Internat. J. Algebra Comput, 9(2), 157-168, 1999

[10] R. Willard, New tools for proving dualizability, Dualities, Interpretability and Ordered Structures (Lisbon, 1997), J. Vaz de Carvalho and I. Ferreirim, Centro de Algebra da Universidade de Lisboa, 69-74, 1999.

[11] L. Zadori, Natural Duality via a finite set of relations, Bulletin of the Australian Mathematical Society 51, 469-478, 1995.

(W. Bentz) School of Mathematics and Physical Sciences, University of Hull, UK E-mail address, W. Bentz: W.Bentz@hull.ac.uk 
(P. Gillibert) Universidad de Valparaíso, Chile

E-mail address, P. Gillibert: pgillibert@yahoo.fr

$U R L$, P. Gillibert: http://www.gillibert.fr/pierre/

(L. Sequeira) Departamento de Matemática, Faculdade de Ciências da Universidade de Lisboa, Lisbon, Portugal

E-mail address, L. Sequeira: lfsequeira@fc.ul.pt 